\title{
Whole-exome sequencing to identify novel somatic mutations in squamous cell lung cancers
}

\author{
CUI-XIA ZHENG ${ }^{1 *}$, ZHAO-HUI GU ${ }^{2 *}$, BING HAN $^{3 *}$, RONG-XIN ZHANG $^{4}$, CHUN-MING PAN $^{5}$, \\ YI XIANG $^{1}$, XIA-JUN RONG ${ }^{1}$, XIA CHEN $^{5}$, QING-YUN LI ${ }^{1}$ and HUAN-YING WAN ${ }^{1}$ \\ ${ }^{1}$ Department of Respiration, Ruijin Hospital Affiliated to Shanghai Jiaotong University School of Medicine, \\ Shanghai 200025; ${ }^{2}$ Shanghai Centre for Systems Biomedicine, Shanghai Jiao Tong University, Shanghai 200240; \\ ${ }^{3}$ Department of Endocrinology, Shanghai Ninth People's Hospital Affiliated to Shanghai Jiaotong University \\ School of Medicine, Shanghai 200011; ${ }^{4}$ Department of Tumor Surgery, the First Hospital Affiliated \\ to Bengbu Medical College, Bengbu 233003; ${ }^{5}$ State Key Laboratory of Medical Genomics, \\ Center of Molecular Medicine, Shanghai Institute of Endocrinology, Ruijin Hospital Affiliated \\ to Shanghai Jiaotong University School of Medicine, Shanghai 200025, P.R. China
}

Received March 25, 2013; Accepted May 8, 2013

DOI: 10.3892/ijo.2013.1991

\begin{abstract}
Squamous cell lung cancer is a major histotype of non-small cell lung cancer (NSCLC) that is distinct from lung adenocarcinoma. We used whole-exome sequencing to identify novel non-synonymous somatic mutations in squamous cell lung cancer. We identified 101 single-nucleotide variants (SNVs) including 77 non-synonymous SNVs (67 missense and 10 nonsense mutations) and 11 INDELs causing frameshifts. We also found four SNVs located within splicing sites. We verified 62 of the SNVs (51 missense, 10 nonsense and 1 splicing-site mutation) and 10 of the INDELs as somatic mutations in lung cancer tissue. Sixteen of the mutated genes were also mutated in at least one patient with a different type of lung cancer in the Catalogue of Somatic Mutation in Cancer (COSMIC) database. Four genes (LPHN2, TP53, MYH2 and TGM2) were mutated in approximately $10 \%$ of the samples in the COSMIC database. We identified two missense mutations in C10orf137 and MS4A3 that also occurred in other solid-tumor tissues in the COSMIC database. We found another somatic mutation in EP300 that was mutated in $4.2 \%$ of the 2,020 solid-tumor samples in the COSMIC database. Taken together, our results implicate TP53, EP300, LPHN2, C10orf137, MYH2, TGM2 and MS4A3 as potential driver genes of squamous cell lung cancer.
\end{abstract}

Correspondence to: Dr Huan-Ying Wan, Department of Respiration, Ruijin Hospital Affiliated to Shanghai Jiaotong University School of Medicine, 197 Ruijin Rd. II, Shanghai 200025, P.R. China

E-mail: hy_wan2013@163.com

*Contributed equally

Key words: lung cancer, non-small cell, whole-exome sequencing, somatic mutation

\section{Introduction}

Lung cancer is the leading cause of cancer-related death worldwide and accounts for one quarter of all cancer mortalities in the US (1). Non-small cell lung cancer (NSCLC) accounts for approximately $80 \%$ of all lung cancer cases and can be classified by histotypes as adenocarcinoma (AC), squamous cell carcinoma (SCC), and large-cell lung cancer (LCLC). The high mortality rate of lung cancer is mainly attributed to the disease not being diagnosed until it is in advanced stages. Chemotherapy with platinum-based drugs in combination with taxanes, camptothecins, or vinca alkaloids, the first-line treatment for patients with NSCLC, has made little progress in improving prognoses in recent decades (1).

Similar to other malignancies, tumorigenesis in NSCLC depends on the clustering of gene dysfunction as a result of genetic susceptibility and/or the accumulation of noxious environmental factors. The discoveries of recurrent mutations in the epidermal growth-factor receptor (EGFR) kinase and fusions, such as $E M L 4-A L K$, involving anaplastic lymphoma kinase (ALK) led to a dramatic change in the treatment of lung AC $(2,3)$. Recent data suggest that substance CI1040 can bind to MEK and mutated BRAF, resulting in the shrinkage of lung ACs that harbor mutated $K R A S$ and $B R A F$, respectively (4). Other recent data show that targeting mutations in $A K T 1, E R B B 2$ and PIK3CA and fusions involving ROSI and RET may also be successful (5). Unfortunately, activating mutations in $E G F R$, EML4-ALK fusions, and mutations in KRAS are only detected in lung $\mathrm{AC}$, and are not present in the second most-common type of lung cancer, SCC (6). Thus, targeted agents developed for lung AC are largely ineffective against lung SCC (7).

Lung SCC accounts for $45 \%$ of NSCLC, and is therefore a main cause of lung cancer mortality. Lung SCC is different from $\mathrm{AC}$ in terms of its clinical features, response to therapies, and, most importantly, its genetic-variation profiles. Research on the molecular mechanisms of lung SCC is limited with few encouraging outcomes. Previous candidate-gene studies 
of lung SCC reported recurring mutations in several genes including TP53, NFE2L2, KEAP1, BAI3, FBXW7, GRM8, MUC16, RUNXIT1, STK11 and ERBB4 (8,9). Other recent data showed that lung SCC with FGFRI amplification and DDRI mutations would be responsive to targeted agents (10-12).

We performed whole-exome sequencing of lung SCC tissue and adjacent normal lung tissue from one patient to identify new mutations involved in lung SCC tumorigenesis. We annotated our results by comparing them with those of previous matched tumor/normal sequencing studies in the Catalogue of Somatic Mutation in Cancer (COSMIC) database.

\section{Materials and methods}

Sample collection and DNA extraction. We obtained 98 paired tumor-tissue and adjacent normal-tissue samples including 44 lung SCCs, 49 lung ACs, and 5 LCLCs from patients diagnosed with NSCLC who underwent definitive surgical resection prior to receiving chemotherapy or radiation at the First Hospital Affiliated to Bengbu Medical College or at Ruijin Hospital Affiliated to Shanghai Jiaotong University School of Medicine. The Ethics Committee of Ruijin Hospital approved the study and we also provided written informed consent. We performed all our experiments according to the Helsinki Declaration. We conducted a pathology review of each sample to establish a histologic diagnosis. The median age of the patients was 53 years (range 27-83). We extracted genomic DNA from the tissue samples using the Automatic Nucleic Acid Isolation System (QuickGene-610L, Fujifilm Life Science, Tokyo, Japan). We selected tumor and adjacent normal-tissue samples from one 55-year-old male patient with lung SCC for whole-exome sequencing.

Targeted sequence capture. We captured the genomic DNA on a NimbleGen 2.1M human-exome array according to the manufacturer's protocols (Roche/NimbleGen). We aimed to capture most of the human exome from the DNA sample with the NimbleGen chip, which contains $24 \mathrm{Mb}$ CCDS ( $\sim 85 \%$ of the US National Center for Biotechnology Information CCDS Database) region across approximately 17,000 genes in $34 \mathrm{Mb}$ targeted nucleotides. The DNA was sheared by sonication and the adaptors ligated to the fragments. The adaptor-ligated templates were fractioned by agarose-gel electrophoresis and the fragments were excised to the desired size. We hybridized the extracted DNA to the capture array at $42^{\circ} \mathrm{C}$ using the manufacturer's buffer. The array was washed twice at $47.5^{\circ} \mathrm{C}$ and three more times at room temperature $\left(20-25^{\circ} \mathrm{C}\right)$ with the manufacturer's buffers. The bound genomic DNA was eluted in $125 \mathrm{mM} \mathrm{NaOH}$ for $10 \mathrm{~min}$ at room temperature. The selected DNA fragments were amplified by ligation-mediated PCR, purified and sequenced on the Illumina platform.

The single-nucleotide variants (SNVs) and INDELs discovered by the whole-exome sequencing was confirmed by sequencing the PCR amplification with specific primers on ABI3703 (data no shown).

Alignment, SNV/INDEL calling and quality control. We aligned the paired-end reads to the reference human genome (hg19, http://genome.ucsc.edu/) using third-party software, BWA, with the default parameters. The average sequencing depth of the case and control samples was more than 50X, and the coverage of the target area was approximately $80 \%$ (data no shown). Approximately $70 \%$ of the nucleotides within the coding region were covered by at least 10 different reads.

We re-aligned the INDEL regions of the bam file using GATK software (version 1.1-30). The SNVs and INDELs were extracted using the unified genotyper function in accordance with the default parameters. To call an SNV or an INDEL, the mapping quality had to be no less than 40 , the mutation had to be measured at least five times, and the allelic heterozygosity had to be $>12.5 \%$.

Mutation annotation based on COSMIC. We confirmed the mutations by ABI 3730 sequencing and annotated them using the COSMIC database. The latest version of the COSMIC database contains 14,819 articles on tumor somatic-mutation research, including 2,556 whole-genome sequencing studies of tumor tissues which scanned 22,170 genes for mutations, and a total of 773,098 tissue samples. The database contains 405,271 mutation sites with 224,649 single-site mutations (there is no reproducible variation in the tumor samples), 8,931 fusion-gene variants, and 7,503 genomic rearrangements.

Molecular modeling of TP53. To further investigate the influence of the R249S mutation on the TP53 structure, a three-dimensional computer model was constructed with the NOC program. TP53 (residues 219-292) was modeled with the SWISS-MODEL software (http://swiss model.expasy.org/) using the crystal structure of human TP53 (PDB accession code 2qxa, chain B) as a template (13).

Validation of sequencing results. Four genes (EP300, CADM2, $C E P 63$ and $M A P 3 K 3$ ) that harbored amino acid replacements in the whole-exome sequencing sample were selected based on their functions and sequenced their exons and exon/intron junctions in the 98 paired lung cancer/normal tissue samples. EP300 and MAP3K3 are, respectively, involved in the TP53 and RAS singnal pathway, which plays an important role in the pathogenesis of lung cancer. CEP63 binds to and recruits Cdk1 to centrosomes, and thus regulates mitotic entry (14). Although the function of $C A D M 2$ remained elusive, it has been reported that CADM2 was recurrently disrupted in prostate cancer (15).

\section{Results}

Identification of somatic mutations from lung SCC. By comparing the whole-exome sequencing data between the tumor and normal lung tissues from a single patient with lung SCC, we identified 293 somatic SNVs and 62 INDELs (29 deletions and 33 insertions) (Fig. 1). The majority of the SNVs were located in inter-genic regions or introns. We identified 101 SNVs, including 77 non-synonymous SNVs (67 missense mutations and 10 nonsense mutations) and 11 INDELs, in the coding regions of genes (data no shown). We also found four SNVs in splicing sites (within three nucleotides of a splicing adaptor or receptor) (data no shown).

Confirmation of the somatic non-synonymous variants in lung SCC. We designed specific primers to verify the 77 nonsynonymous SNVs, 11 INDELS, and 4 splicing-site mutations 


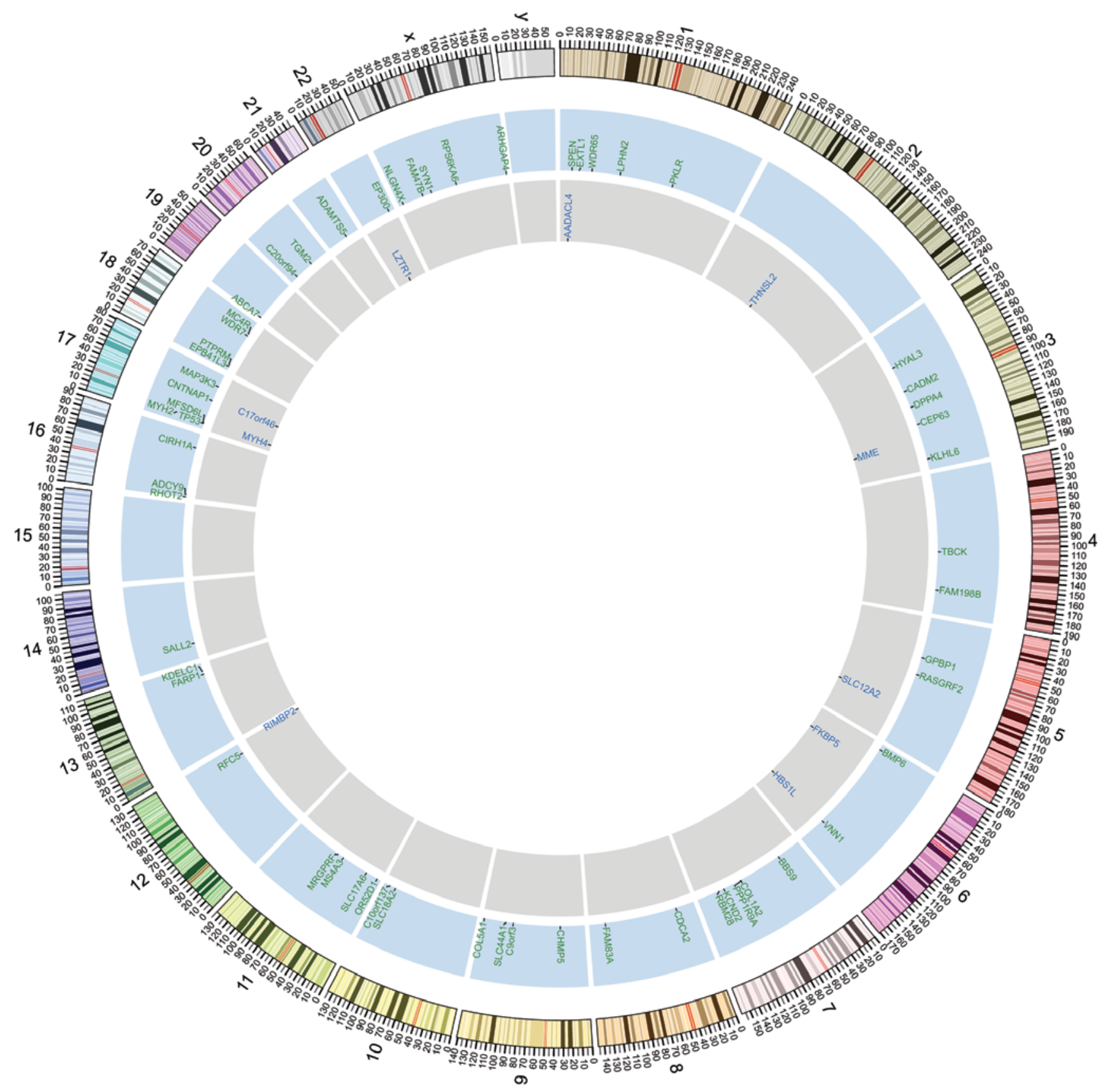

Figure 1. Non-synonymous somatic mutations discovered by whole-exome sequencing. From outside to inside: the colored ring and number represent chromosome number and partition; the blue ring and letters indicate genes with non-synonymous SNVs and their corresponding chromosomes; the gray ring shows non-synonymous INDELs and their corresponding chromosomes.

by sequencing on ABI3730. We confirmed 51 missense mutations, 10 nonsense mutations and 1 splicing-site mutation to be somatic mutations in the lung SCC tissue by ABI 3730 sequencing (Table I). We also verified 10 of the 11 INDELs as somatic mutations in lung SCC tissue (Table I and Fig. 1).

We further analyzed the 51 confirmed missense mutations by searching the SIFT database (16) to predict their effects on protein structure. We found that 15 of the missense mutations could dramatically affect protein functions (Table I).

Comparison with COSMIC database. In the samples of lung cancer tissues and other types of solid tumors in the COSMIC database, we found previously identified mutations in all of the genes, except for MRGPRF, containing the $62 \mathrm{SNVs}$ and 10 INDELs confirmed in our study by ABI 3730 sequencing. Fifteen of the genes with non-synonymous SNVs and one with an INDEL (LZTRI) had previously identified mutations in at least one sample of different pathological types of lung cancer; four of those genes (LPHN2, TP53, MYH2 and TGM2) were mutated in close to or more than $10 \%$ of the tumor tissues available in the COSMIC database. TP53 and LPHN2 were sequenced in more than 500 tumor samples, and their mutation frequencies were $18.3 \%(12,142 / 66,304)$ and $8.32 \%(49 / 589)$, respectively (Table II). TP53, a well-known oncogene that plays 


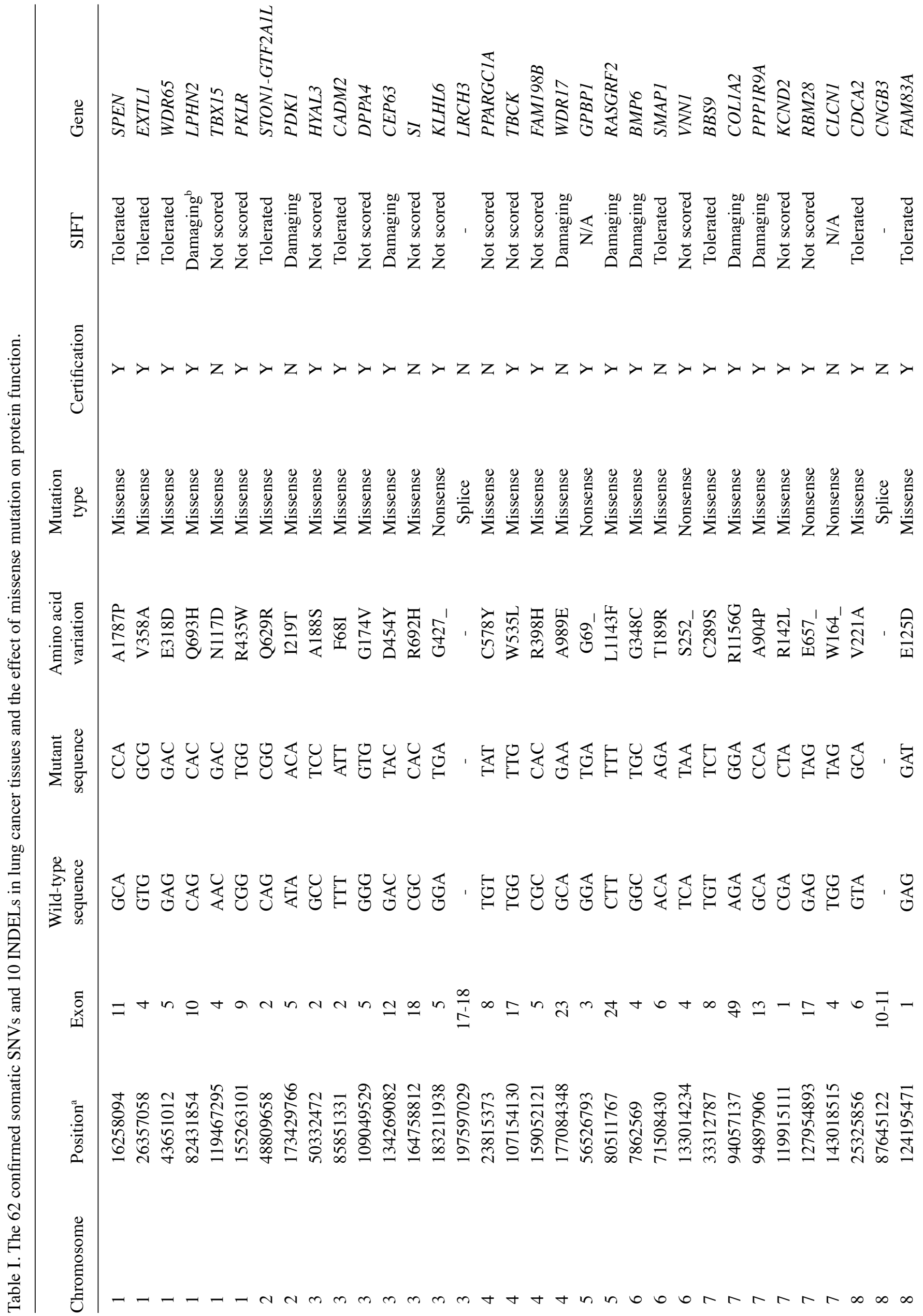


랄

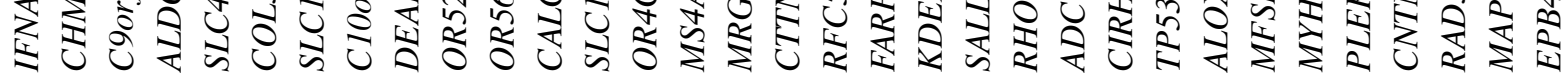

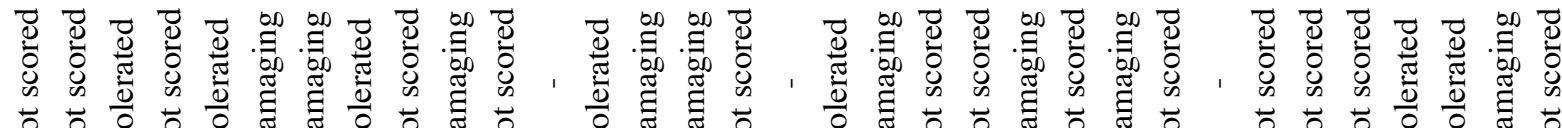

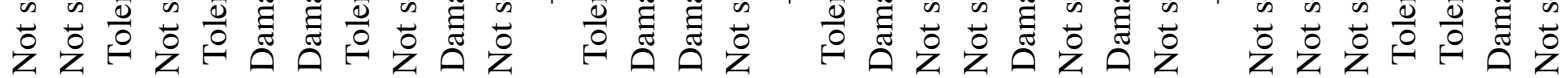

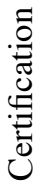

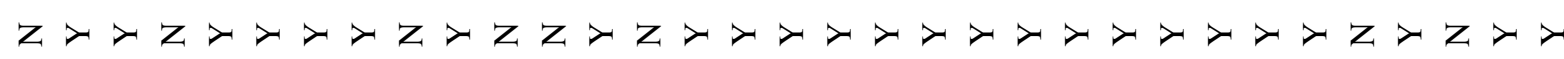

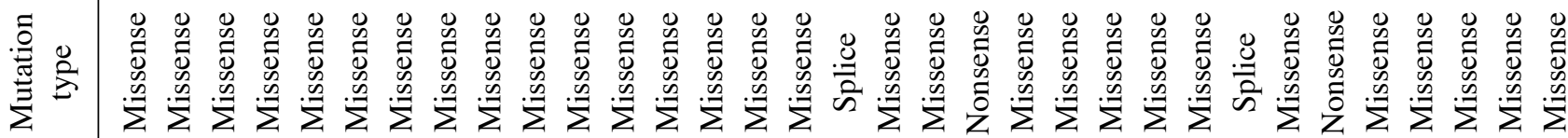

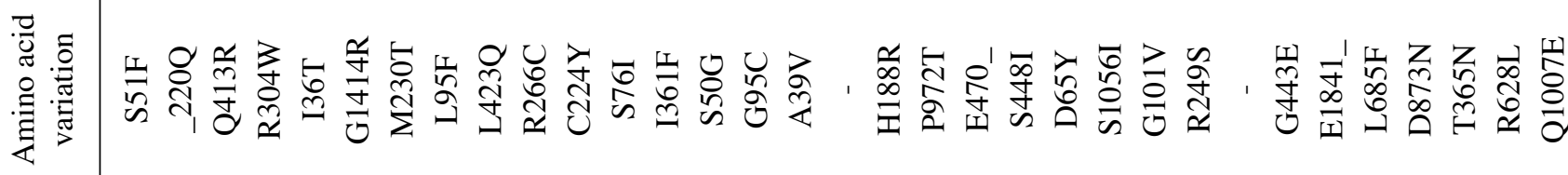

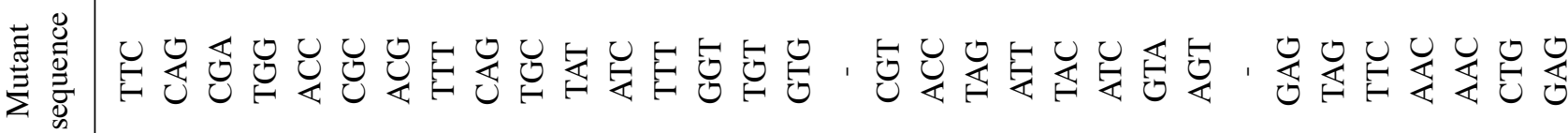

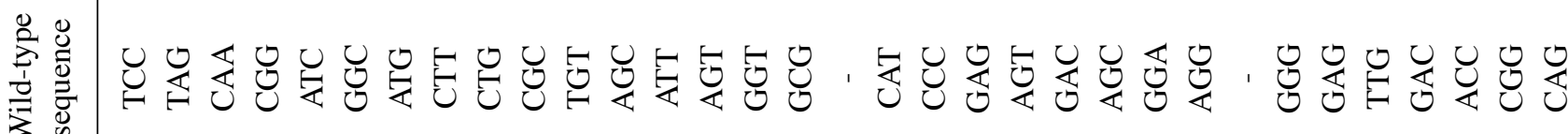

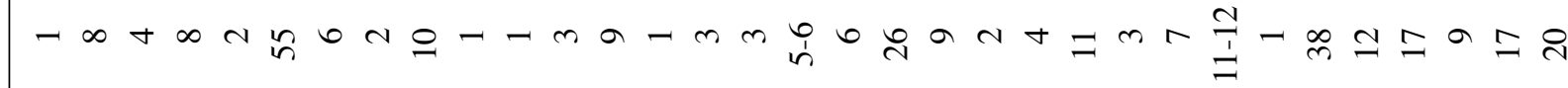

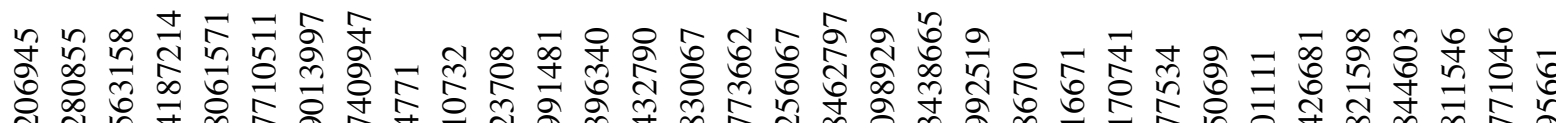

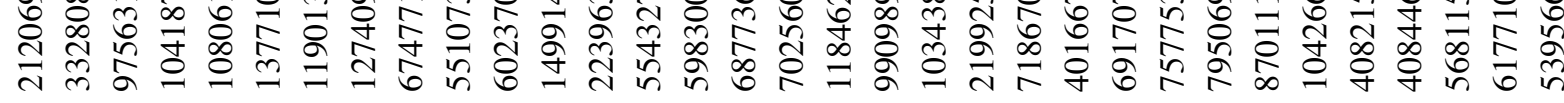




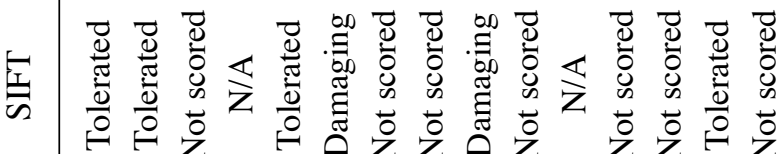

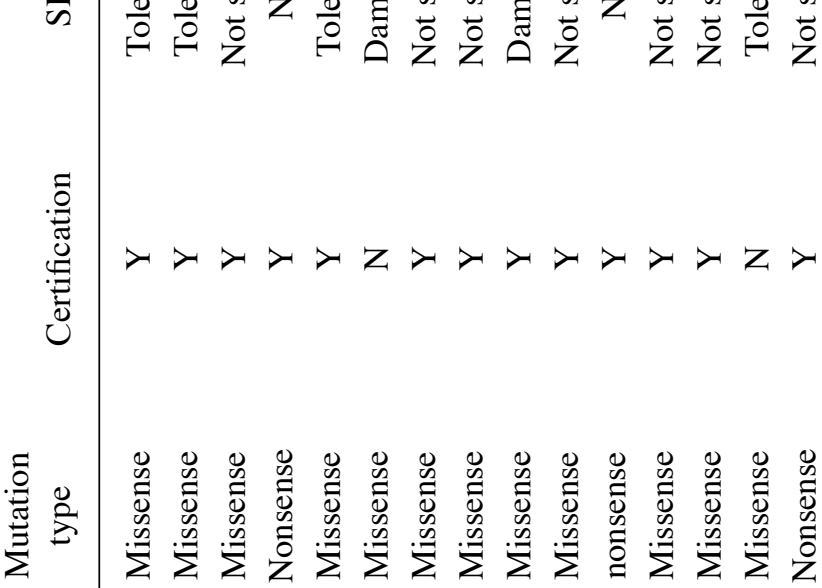

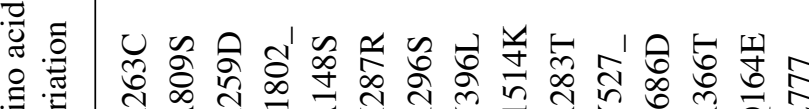

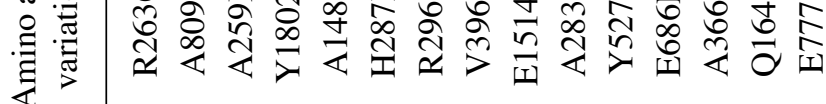

穿总

龸苞

文

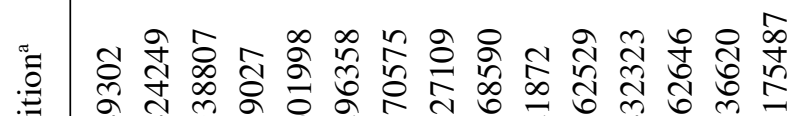
:

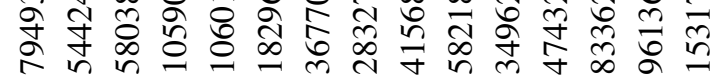

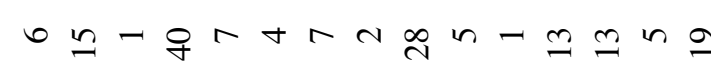

$:$
$\vdots$
$\vdots$
$\vdots$
$\vdots$
$\vdots$

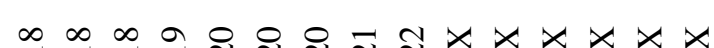

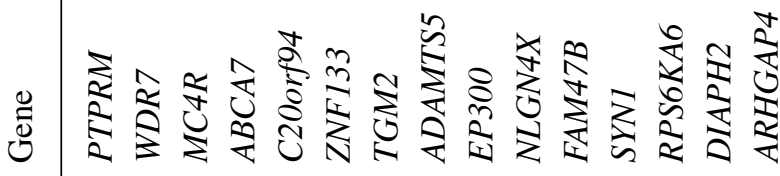

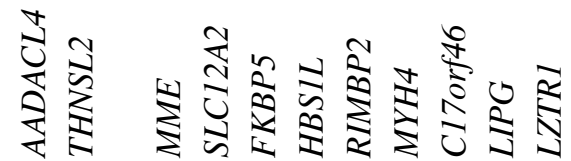

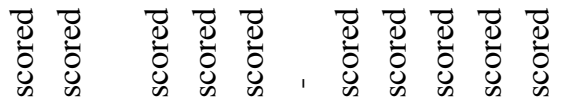

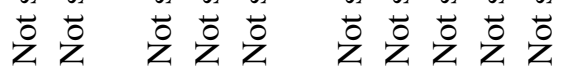

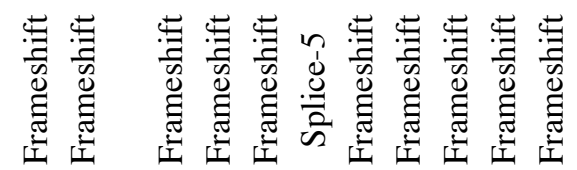

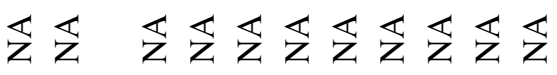

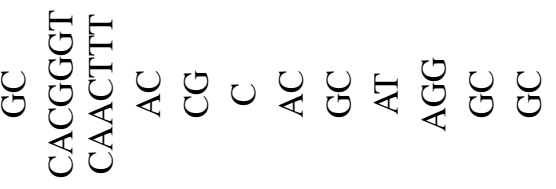

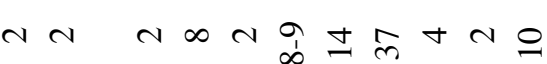

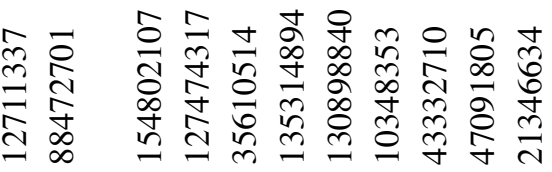

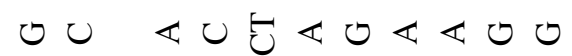

$-4$

$m$ in 0 on $n-\infty \mathrm{N}$ 
Table II. Genes with reoccurring mutations in lung cancer tissues in the COSMIC database.

\begin{tabular}{llccccc}
\hline Chromosome & Gene & ACC & SCC & SCLC & Solid tumors & Hematol. cancer \\
\hline 1 & SPEN & - & $1 / 11$ & - & - & - \\
1 & LPHN2 & $1 / 57$ & $2 / 63$ & - & $44 / 465$ & $2 / 4$ \\
2 & PDK1 & $1 / 253$ & $2 / 70$ & - & $4 / 586$ & - \\
3 & CADM2 & - & $1 / 10$ & - & $10 / 304$ & $2 / 2$ \\
4 & PPARGC1A & - & $2 / 10$ & - & $14 / 308$ & - \\
10 & ClOorf137 & $1 / 57$ & $1 / 63$ & - & $22 / 557$ & - \\
11 & MS4A3 & 1 & - & - & $6 / 96$ & - \\
17 & TP53 & $952 / 1,386$ & $452 / 866$ & - & $8,756 / 58,462$ & $1,982 / 5,590$ \\
17 & MYH2 & - & & $1 / 1$ & $36 / 218$ & - \\
17 & CNTNAP1 & - & $1 / 63$ & - & $19 / 404$ & - \\
18 & $M C 4 R$ & - & $1 / 63$ & - & $4 / 392$ & - \\
20 & TGM2 & - & - & $1 / 1$ & $13 / 166$ & $28 / 525$ \\
22 & EP300 & - & $1 / 63$ & - & $57 / 1,495$ & - \\
$\mathrm{X}$ & RPS6KA6 & & $1 / 16$ in LCC & & $9 / 292$ & - \\
$\mathrm{X}$ & DIAPH2 & $1 / 1$ & - & - & $18 / 132$ & - \\
22 & LZTR1 & $2 / 188$ & - & - & $14 / 104$ & - \\
\hline
\end{tabular}

Results represent positive cases/total cases. SCC, squamous cell carcinoma; ACC, adenocarcinoma; LCC, large-cell cancer; SCLC, small cell lung cancer; Hematol, hematological.

an important role in lung cancer pathogenesis, is mutated in $62.3 \%(1,404 / 2,252)$ of the lung cancer tissue samples in the COSMIC database.

We also identified two missense mutations in C10orf137 and $M S 4 A 3$, respectively, that also appear in different lung cancer tissues in the COSMIC database. It is worth noting that the mutation in C10orf137 was investigated in more than 500 solid tumor tissues and its frequency is approximately $3.55 \%$ (24/677) in COSMIC database (Table II). We identified a somatic mutation in EP3OO and found that $4.2 \%(85 / 2,020)$ of the tumor tissues in the COSMIC database also had mutations in EP300 (Table II).

Based on the comparisons of our whole-exome sequencing results with the previously identified mutations in the COSMIC database, we identified seven genes ( $L P H N 2, T P 53, M Y H 2$, TGM2, C10orf137, EP300 and MS4A3) as possible drivers of lung cancer pathogenesis (Table II and Fig. 2).

Computer modeling and analysis of TP53. Our study is the first, however, to identify a $\mathrm{C}>\mathrm{A}$ substitution in squamous cell lung cancer tissue changing Arg to Ser at amino acid position 249 (R249S) in TP53. By molecular modeling, a charged basic amino acid (Arg) was replaced by an neutral amino acid (Ser) at codon 249, which caused an abnormal electrostatic-charge distribution in the DNA-binding domain of TP53 (Fig. 3).

Validation of sequencing results. We sequenced all of the exons of four genes (MAP3K3, CEP63, CADM2 and EP300) in 98 additional lung cancer samples; including 44 lung SCCs, 49 ACs, and 5 LCLCs; and found no mutations in the coding regions. We found a deletion of 2-4 cytosine residues in the 5'UTR of CEP63 in three of the samples, but the mutations did not change the protein sequences. We also identified a $\mathrm{C}>\mathrm{G}$ variant located at nucleotide position 3207 of MAP3K3 (NM_2033351) in one patient; the variant was located in the 3'UTR, but did not change the protein sequence.

\section{Discussion}

We used whole-exome sequencing to identify 72 somatic mutations, including 62 SNVs (51 missense mutations, 10 nonsense mutations, and 1 splicing-site mutation) and 10 INDELs, in the coding regions of different genes from a single case of lung SCC. We found somatic mutations in 71 of the genes in at least one additional tumor sample in the COSMIC database. We found mutations in 16 of the genes in at least one additional lung cancer patient. Four genes (LPHN2, TP53, MYH2 and TGM2) were mutated in approximately $10 \%$ of the tumor samples in the COSMIC database.

We found the most mutations in TP53: $68.7 \%(952 / 1,386)$ of lung AD cases and 52.2\% (452/866) of lung SCC cases. Although TP53 is frequently mutated in tumor tissues from patients with lung cancer, our study is the first to describe the R249S somatic missense mutation in lung cancer tissues. SIFT analysis showed that the R249S mutation in TP53 could dramatically influence the structure of the TP53 protein (16). It worth noting that the R249S mutation in TP53 is frequently found in HBV-induced hepatic-cell carcinoma, accounting for $90 \%$ of the TP53 mutations identified in liver cancer (17). In hepatocellular-carcinoma cell lines, the R249S mutation abolishes the capacity for TP53 to bind p53 response elements and trans-activate p53 target genes. Moreover, in a p53-null Hep3B cell line that constitutively expresses both the R249S variant of TP53 and the hepatitis-B virus antigen HBx 
A

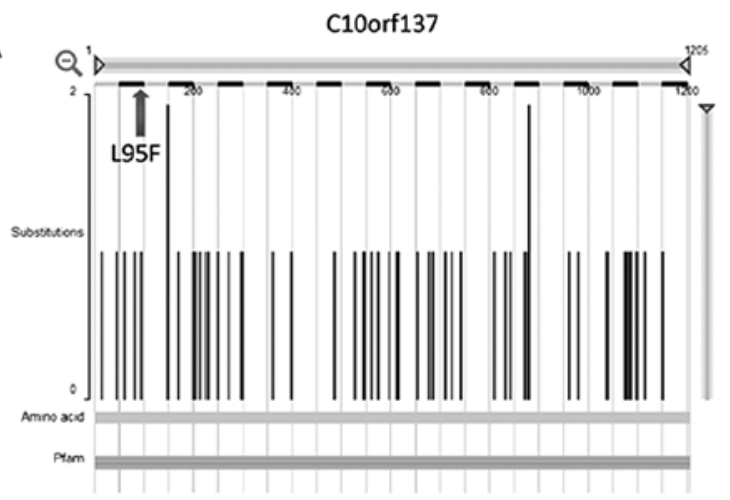

C

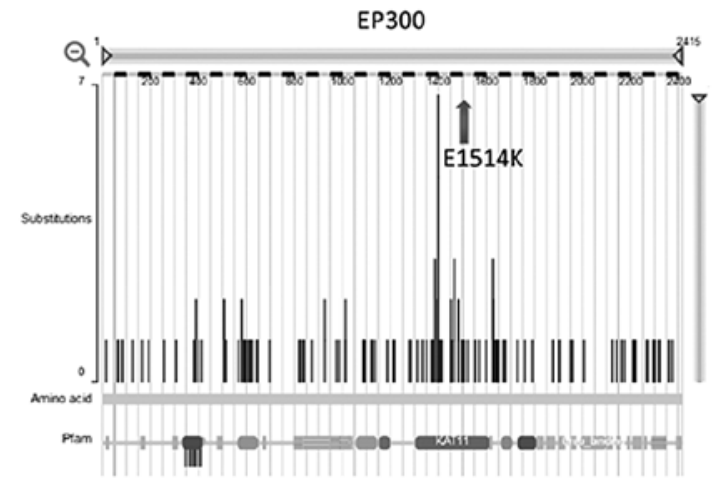

$\mathrm{E}$

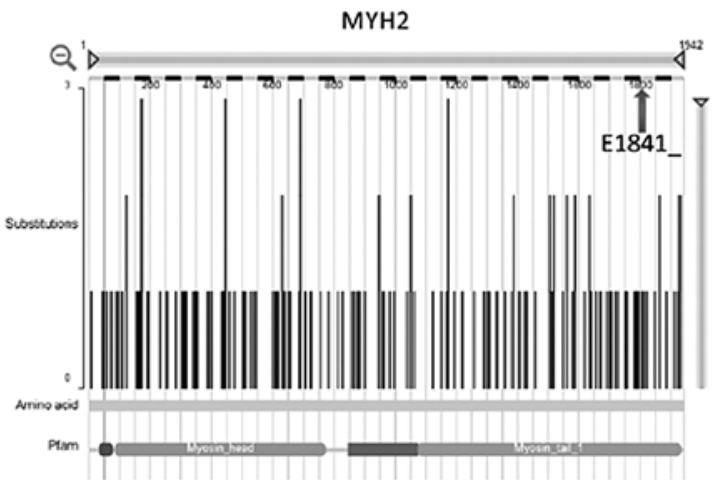

G

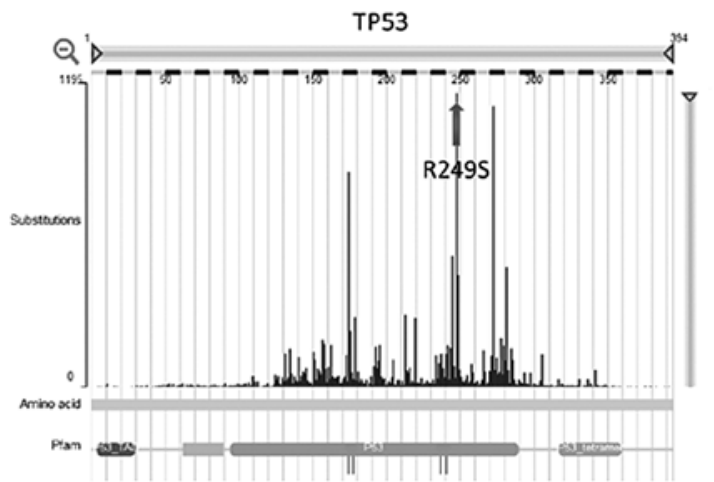

B
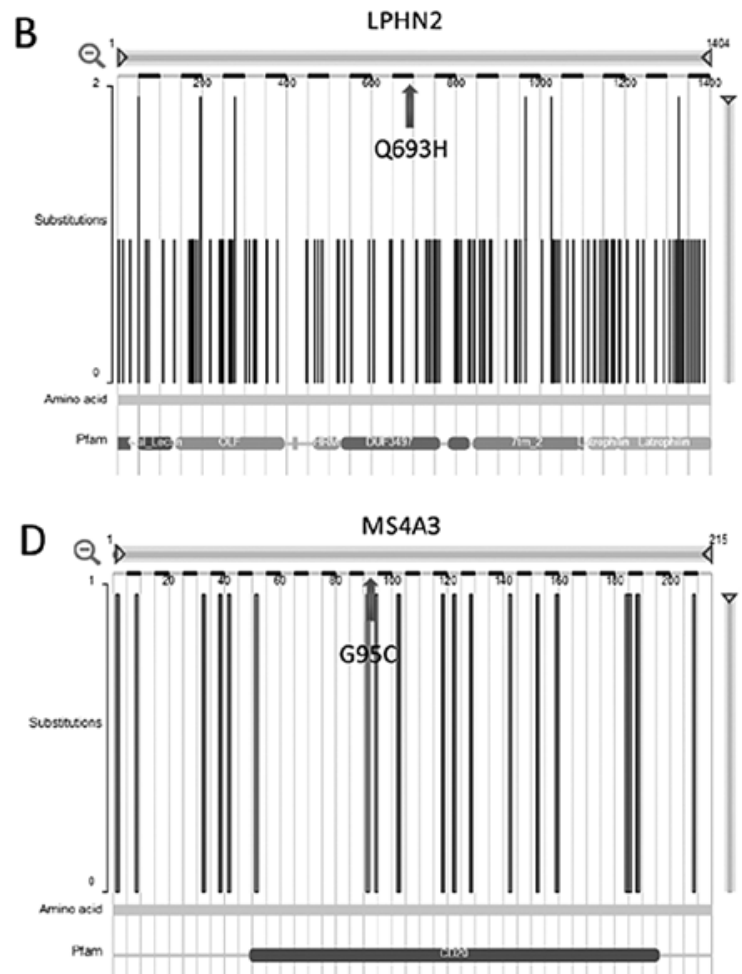

$\mathrm{F}$

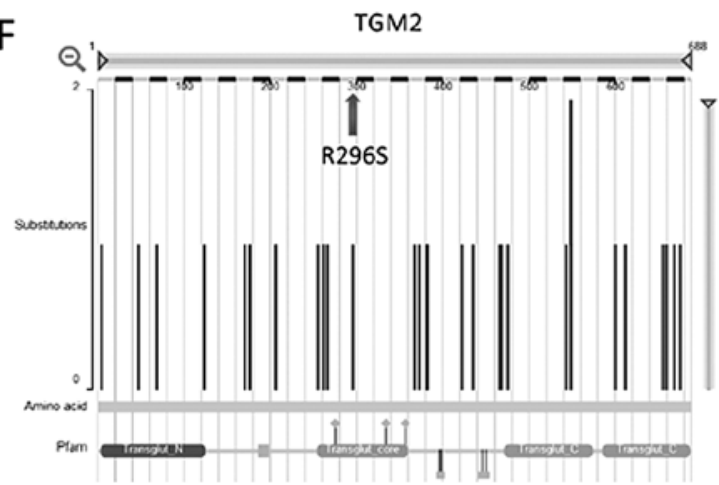

Figure 2. The mutation frequency of seven genes. Arrows indicate the distinct mutation sites discovered in our investigation. TP53 has a mutation 'hot spot' in DNA-binding domain.

(PLC/PRF/5), the silencing of either R249S TP53 or HBx by RNA interference inhibited cellular proliferation, but without additive effects when both genes were silenced (17). Taken together with the previous results, our results suggest that the R249S mutation in TP53 may be play a key role in lung cancer pathogenesis.
LPHN2 was previously sequenced in more than 500 tumor samples and found to be mutated in $9.46 \%$ (44/465) of solid-tumor samples. Moreover, somatic mutations in LPHN2 occurred in $3.17 \%$ of lung SCC (2/63) samples in the COSMIC database. LPHN2 encodes a member of the latrophilin subfamily of G-protein coupled receptors (GPCR), 

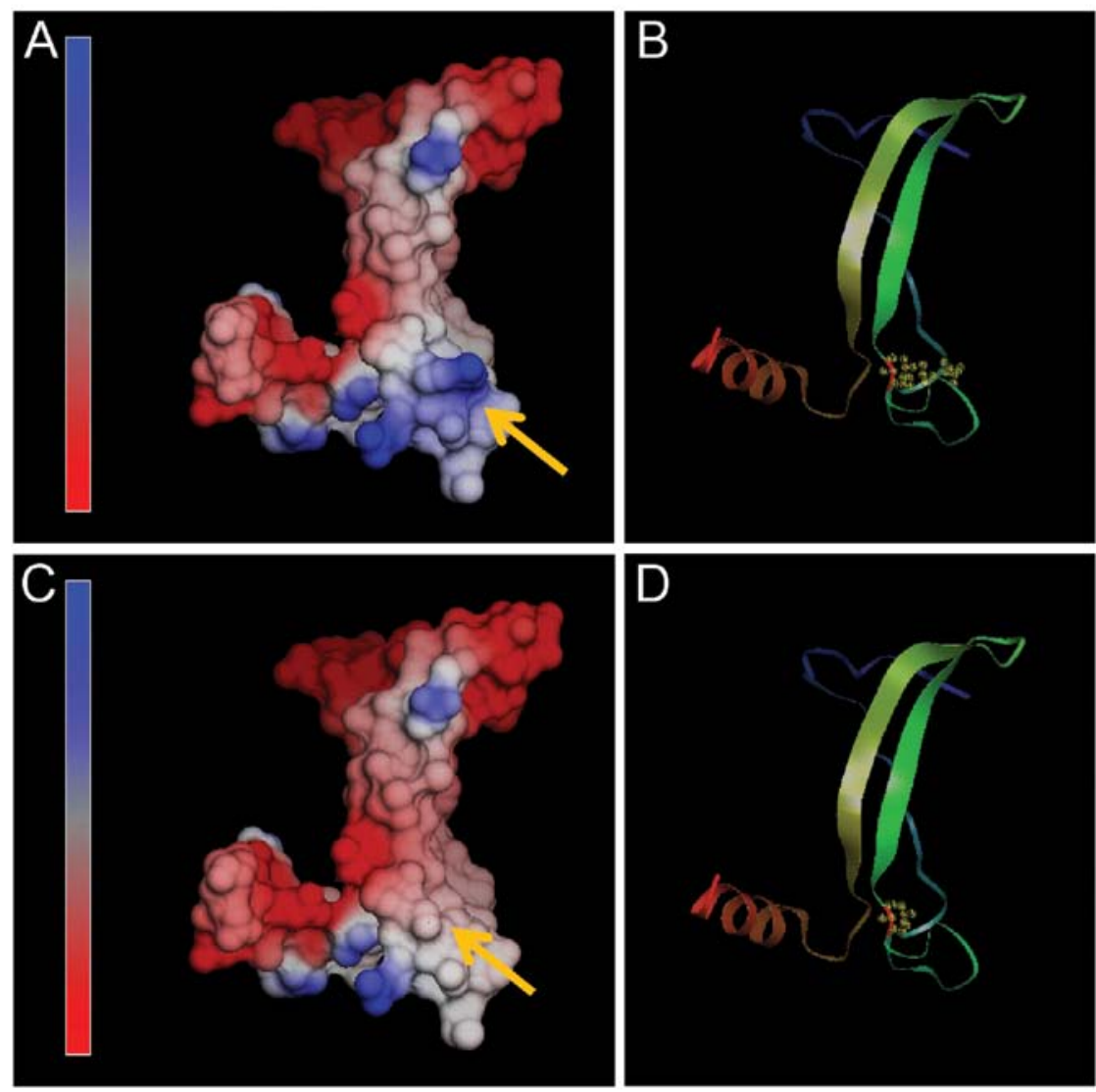

Figure 3. The effect of the novel somatic mutation (R490S) on the structure and function of TP53. (A and B) Structure of wild-type TP53. (C and D) Structure of mutated TP53. The arrow indicates the mutated amino acid. Red represents positively charged area. Blue represents negatively charged area.

and genome-wide association analysis found a significant association between SNVs of LPHN2 and paclitaxel sensitivity in NCI60 cancer cell lines (18).

MYH2 and TGM2 were mutated in $16.5 \%(36 / 218)$ and $7.8 \%$ (13/166) of solid tumors; neither mutation, however, was previously investigated in SCC samples.

We identified two missense mutations in C10orf137 and $M S 4 A 3$, respectively; both were previously identified in different lung cancer tissues. Mutations in C10orf137 were previously investigated in more than 500 solid-tumor samples and found in $3.55 \%$ (24/677) of the samples. Recently, Gylfe et al identified one missense germ-line mutation in C10orf137 in 45 familial patients with colorectal cancers, while none of the 890 population-matched healthy controls had the same mutation (19). The function of C10orf137, however, is still unknown. MS4A is a member of the fourtransmembrane protein family. MS4A proteins execute diverse functions, acting as cell-surface signaling molecules and intracellular adapter proteins. Tissue microarray analysis showed MS4A3 expression in a wide variety of ACs including breast, prostate, and ovarian cancers (20). Moreover, previous studies showed that MS4A3 forms a functionally relevant complex with cyclin-dependent kinase-associated phosphatase and CDK2 (21), suggesting that MS4S3 may be a novel modulator of the cell cycle. Further study is needed to explain the role of MS4A3 in lung cancer pathogenesis.
We identified a somatic mutation in EP300 that was previously identified in $4.2 \%(85 / 2,020)$ of the tumor samples in the COSMIC database. Recurrent mutations clustered around the histone acetyltransferase domain in EP300 were recently described in small-cell lung cancers (22). EP300 plays an important role in cell proliferation and differentiation by regulating gene transcription via chromatin remodeling (23-25). EP300 is also an important modulator of the TP53 signaling pathway; it helps to maintain TP53 stability by regulating the ubiquitination and degradation of TP53 through both MDM2-dependent and MDM2-independent mechanisms $(26,27)$. Moreover, EP300 is required for the TP53-mediated transactivation of target genes because of its co-activator function and its acetylation of histones (28-30). Together with the previous results, our data suggest that EP300 may be a driver gene in lung cancer tumorigenesis.

Several recent whole-genome or exome-sequencing studies aimed at characterizing the genomic and epigenomic landscapes of different histopathological types of lung cancer (ACC, SCC and small-cell cancer) (31-34). The results included a large number and variety of DNA alterations with a mean of more than 150 exonic non-synonymous mutations per lung cancer type (31-34). Analyses by different algorithms identified some genes with significantly elevated mutational frequencies in different histological types of lung cancer $(\mathrm{P}<0.05$; false-discovery rate $\leq 0.1)$. Among these genes, TP53 
was confirmed as a tumorigenesis gene and had the highest mutational frequency (29-81\%) in all of the independent studies. Four other genes; EGFR, KRAS, KEAPI and RB1; were also implicated as important tumorigenesis genes in two independent studies (31-34). A somatic mutation in KEAPI was repeatedly identified in independent studies performed on cohorts of lung SCCs and lung ACs $(31,32)$. Somatic mutations in $R B 1$ were confirmed in patients with SCLC and lung SCCs $(31,33)$. Most of the significantly mutated genes, however, were identified in only one cohort (31-34). These results suggest that genomic variants in lung cancer tissues are complex; the somatic mutations in distinct genes underscore the differences between subgroups of lung cancer, even within a single histological type. More whole-exome sequencing studies with large sample sizes are needed to increase the available somaticmutation data.

In summary, our results show that whole-exome sequencing is an effective way to detect novel mutations related to lung cancer. Our study indicates seven genes, TP53, EP300, LPHN2, C10orf137, MYH2, TGM2 and MS4A3, that may be drivers of lung cancer tumorigenesis.

\section{Acknowledgements}

We thank all the patients who participated in this study. This study was supported in part by the National Natural Science Foundation of China (81071925 and 30900503) and the Shanghai Science and Technology Committee (10ZR1418300).

\section{References}

1. Borczuk AC, Toonkel RL and Powell CA: Genomics of lung cancer. Proc Am Thorac Soc 6: 152-158, 2009.

2. Satouchi M, Negoro S, Funada Y, et al: Predictive factors associated with prolonged survival in patients with advanced non-small-cell lung cancer (NSCLC) treated with gefitinib. Br J Cancer 96: 1191-1196, 2007.

3. Shaw AT, Yeap BY, Mino-Kenudson M, et al: Clinical features and outcome of patients with non-small-cell lung cancer who harbor EML4-ALK. J Clin Oncol 27: 4247-4253, 2009.

4. Ji H, Wang Z, Perera SA, et al: Mutations in BRAF and KRAS converge on activation of the mitogen-activated protein kinase pathway in lung cancer mouse models. Cancer Res 67: 4933-4939, 2007.

5. Felip E, Gridelli C, Baas P, Rosell R, Stahel R and Panel Members: Metastatic non-small-cell lung cancer: consensus on pathology and molecular tests, first-line, second-line, and thirdline therapy. Ann Oncol 22: 1507-1519, 2011.

6. Rekhtman N, Paik PK, Arcila ME, et al: Clarifying the spectrum of driver oncogene mutations in biomarker-verified squamous carcinoma of lung: lack of EGFR/KRAS and presence of PIK3CA/AKT1 mutations. Clin Cancer Res 18: 1167-1176, 2012.

7. James J, Ruggeri B, Armstrong RC, et al: CEP-32496: a novel orally active BRAF(V600E) inhibitor with selective cellular and in vivo antitumor activity. Mol Cancer Ther 11: 930-941, 2012.

8. Shibata T, Ohta T, Tong KI, Kokubu A, Odogawa R, Tsuta K, Asamura H, Yamamoto $M$ and Hirohashi S: Cancer relatedmutations in NRF2 impair its recognition by Keap1-Cul3 E3 ligase and promote malignancy. Proc Natl Acad Sci USA 105: $13568-13573,2008$.

9. Kan Z, Jaiswal BS, Stinson J, et al: Diverse somatic mutation patterns and pathway alterations in human cancers. Nature 466: $869-873,2010$

10. Dutt A, Ramos AH, Hammerman PS, et al: Inhibitor-sensitive FGFR1 amplification in human non-small cell lung cancer. PLoS One 6: e20351, 2011.

11. Hammerman PS, Sos ML, Ramos AH, et al: Mutations in the DDR2 kinase gene identify a novel therapeutic target in squamous cell lung cancer. Cancer Discov 1: 78-89, 2011.
12. Weiss J, Sos ML, Seidel D, et al: Frequent and focal FGFR1 amplification associates with therapeutically tractable FGFR 1 dependency in squamous cell lung cancer. Sci Transl Med 2: 62ra93, 2010

13. Arnold K, Bordoli L, Kopp J and Schwede T: The SWISSMODEL Workspace: A web-based environment for protein structure homology modeling. Bioinformatics 22: 195-201, 2006.

14. Löffler H, Fechter A, Matuszewska M, et al: Cep63 recruits Cdk1 to the centrosome: implications for regulation of mitotic entry, centrosome amplification, and genome maintenance. Cancer Res 71: 2129-2139, 2011.

15. Berger MF, Lawrence MS, Demichelis F, et al: The genomic complexity of primary human prostate cancer. Nature 470: 214-220, 2011.

16. Ng PC and Henikoff S: SIFT: predicting amino acid changes that affect protein function. Nucleic Acids Res 31: 3812-3814, 2003.

17. Gouas DA, Shi H, Hautefeuille AH, et al: Effects of the TP53 p.R249S mutant on proliferation and clonogenic properties in human hepatocellular carcinoma cell lines: interaction with hepatitis B virus X protein. Carcinogenesis 31: 1475-1482, 2010.

18. Eng L, Ibrahim-Zada I, Jarjanazi H, Savas S, Meschian M, Pritchard KI and Ozcelik H: Bioinformatic analyses identifies novel protein-coding pharmacogenomic markers associated with paclitaxel sensitivity in NCI60 cancer cell lines. BMC Med Genomics 4: 18, 2011.

19. Gylfe AE, Sirkiä J, Ahlsten M, Järvinen H, Mecklin JP, Karhu A and Aaltonen LA: Somatic mutations and germline sequence variants in patients with familial colorectal cancer. Int J Cancer 127: 2974-2980, 2010.

20. Kutok JL, Yang X, Folkerth R and Adra CN: Characterization of the expression of HTm4 (MS4A3), a cell cycle regulator, in human peripheral blood cells and normal and malignant tissues. J Cell Mol Med 15: 86-93, 2011

21. Donato JL, Ko J, Kutok JL, et al: Human HTm4 is a hematopoietic cell cycle regulator. J Clin Invest 109: 51-58, 2002.

22. Peifer M, Fernández-Cuesta L, Sos ML, et al: Integrative genome analyses identify key somatic driver mutations of small-cell lung cancer. Nat Genet 44: 1104-1110, 2012.

23. Ogryzko VV, Schiltz RL, Russanova V, Howard BH and Nakatani Y: The transcriptional coactivators p300 and CBP are histone acetyltransferases. Cell 87: 953-959, 1996.

24. Kawasaki H, Eckner R, Yao TP, Taira K, Chiu R, Livingston DM and Yokoyama KK: Distinct roles of the co-activators p300 and CBP in retinoic-acid-induced F9-cell differentiation. Nature 393: 284-289, 1998.

25. Yao TP, Oh SP, Fuchs M, et al: Gene dosagedependent embryonic development and proliferation defects in mice lacking the transcriptional integrator $\mathrm{p} 300$. Cell 93: 361-372, 1998.

26. Grossman SR, Deato ME, Brignone C, Chan HM, Kung AL, Tagami H, Nakatani Y and Livingston DM: Polyubiquitination of p53 by a ubiquitin ligase activity of p300. Science 300: 342-344, 2003.

27. Grossman SR, Perez M, Kung AL, Joseph M, Mansur C, Xiao ZX, Kumar S, Howley PM and Livingston DM: p300/ MDM2 complexes participate in MDM2-mediated p53 degradation. Mol Cell 2: 405-415, 1998.

28. Lill NL, Grossman SR, Ginsberg D, DeCaprio J and Livingston DM: Binding andmodulation of $\mathrm{p} 53$ by p300/CBP coactivators. Nature 387: 823-827, 1997.

29. Espinosa JM and Emerson BM: Transcriptional regulation by p53 through intrinsic DNA/chromatin binding and site-directed cofactor recruitment. Mol Cell 8: 57-69, 2001.

30. Avantaggiati ML, Ogryzko V, Gardner K, Giordano A, Levine AS and Kelly K: Recruitment of p300/CBP in p53-dependent signal pathways. Cell 89: 1175-1184, 1997.

31. Cancer Genome Atlas Research Network: Comprehensive genomic characterization of squamous cell lung cancers. Nature 489: 519-525, 2012.

32. Imielinski M, Berger AH, Hammerman PS, et al: Mapping the hallmarks of lung adenocarcinoma with massively parallel sequencing. Cell 150: 1107-1120, 2012.

33. Rudin CM, Durinck S, Stawiski EW, et al: Comprehensive genomic analysis identifies SOX2 as a frequently amplified gene in small-cell lung cancer. Nat Genet 44: 1111-1116, 2012.

34. Govindan R, Ding L, Griffith M, et al: Genomic landscape of non-small cell lung cancer in smokers and never-smokers. Cell 150: 1121-1134, 2012. 